\title{
Energy Efficient WSN Using Green Task Based Sensing Scheme
}

${ }^{* 1}$ Dr. D.K.Shedge, ${ }^{2}$ Priyanka Sanjay Patil

${ }^{1,2}$ Department of Electronics Engineering, AISSMS, Institute of Information Technology, Pune, India Email:shedgedk@gmail.com, priyankaspatil5@gmail.com

Received: 24 ${ }^{\text {th }}$ November 2018, Accepted: 13 $^{\text {th }}$ February 2019, Published: $3^{\text {th }}{ }^{\text {June }} 2019$

\begin{abstract}
Wireless sensor networks (WSNs) are widely used to monitor environmental conditions for different purposes with in different locations. However, due to the limited duration of the sensor's energy source, many attempts are made to design energy Efficient WSN. Therefore many outcomes have been presented in literature, such as the adaptation of power, scheduling, sleep and wake up techniques to improve the useful life of the WSN. These techniques are presented separately and shown to achieve some benefits in terms of energy efficiency. In this paper, we present a low-energy efficient design system for WSN that prevents unnecessary wasting of power which in-turn increases the network lifetime. The proposed system reduce the overall power consumption up to $50 \%-70 \%$ approximately as compared to conventional task base scheme which will boost the network lifetime.
\end{abstract}

Keywords

Wireless Sensor Networks, Adaptive Power, Task-Based Sensing, Sleep and Wake-up

\section{Introduction}

The WSN is built of several or thousands nodes, where each node is connected to one sensor. Each such sensor network node has typically several parts such as microcontroller for local data processing, data acquisition for sensing purpose, communication unit for transmitting and receiving data from other connected devices and finally the connection is given to power supply unit. More number of sensors are used greater is the accuracy of the network.

As the sensor node for event monitoring are expected to work for long time without recharging their batteries, sleep scheduling is always used during monitoring process. In WSN to increase the network life time, task based sensing was introduced that prevents the unnecessary wasting of power with various wake-up and sleep techniques that allows the inactive nodes to sleep (M. Balakrishnan et al., 2007; J. Heidemann et al., 2004) And also overcome overall synchronization of network and minimize network traffic. Recently various methodologies have been proposed to determine different sleep times for different applications to enhance the network efficiency (B. Jiang et al., 2013; M. Balakrishnan et al., 2007; W. Ye et al., 2004) Sensor nodes will transmit data only when the critical event occurs. The fig1 below shows the basic event that is generated when sink node detects the event and communicate with other nodes to establish communication.
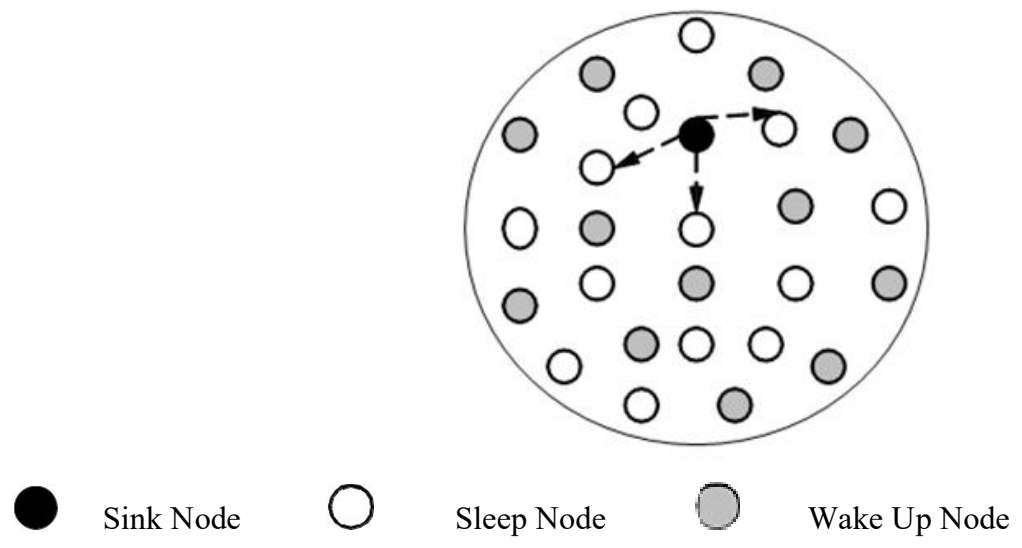

Figure 1: Critical Event Monitoring in WSN.

As the sensor nodes are battery operated it is difficult to replace the batteries of sensor nodes. Therefore energy efficiency is main objective to detect whether sensors energy has been exhausted or not. The nodes communicate to share the collected data to a specified sink node. Where each node has sensing ability depending upon task assigned to it (A. Prayati et al., 2010; I. F. Akyildiz et al., 2002). The main cause of the energy consumption is due to continues listening, collision, interference etc. (A. Tripathi et al., 2015). Therefore 
more research is needed to minimize energy wasted during continuous listening of inactive nodes (A. Prayati et al., 2010). The combination of the task based base sensing and critical event monitoring concept poses a challenge to increase the overall energy efficiency as well as power consumption of overall network. In this paper we propose a smart energy saving algorithm by critical event monitoring and also adaptive power transmission of the network.

The rest of the paper is organized as follows; section II presents the literature survey with various methods and technologies used. Section III presents methodology of system and section IV represents evaluation results of the proposed methodology.

\section{Literature Survey}

This section presents several existing techniques and methodologies used in WSN.

(Xu Xing et al., 2012) investigated about deployment of sensors in 2D region to collect the events. By using Traditional multi-hop routing technique in WSN results in unevenness to report events. To overcome this problem authors developed to maintain the mobility of sink nodes to accommodate the event collection by managing the motion of sink nodes with spatial correlations of events to maximize network lifetime. (N. R. Harris et al., 2008) talks about the states of the charged capacitors evaluated that are based on batteries known as super capacitors for long term deployment of the sensor nodes. And also provided energy stack that hide the complications from application layer to provide direct interface. (K. Phan et al., 2009) proposed power allocation and link scheduling technique by developing cross layer approach that is based on integer linear programming.

The previous studies include two main schemes that is power adaption, sleep and wake up techniques that were analysed in (M. Tahir et al., 2013; D. Son et al., 2004), where the power level was optimized by connection quality and network connectivity. Time in sleep and wake up techniques varies how to sleep randomly, periodically and synchronously. Randomly sleep periods that are established based coverage and network lifetime parameters which are difficult constraints when time of awakening and communication (M. Balakrishnan et al., 2007). Synchronous sleep that is based on virtual clustering requires co-ordination among nodes. But this becomes complicated when large amount of nodes interact with each other (W.

Ye et al., 2004). So to overcome this periodic protocol was designed in which whole burden is on the transmitter predicting the wake up time for the receiver (B. Jiang et al., 2013). This gives the synchronization among the transmitter and receiver to enhance network lifetime. There was also enough work done in tree based routing protocols based on energy efficiency. For example tree based routing technique were introduced in which every node calculate its neighbour nodes and take the path that has max residual energy (S. Charalambos et al., 2012). Minimum number of nodes to transmit these packets are also selected to save energy. Also we have seen that the critical event that is generated based on the task results in energy consumption (A. Tripathi et al., 2015). That means when the critical event monitoring occur then only the slaves will send the data to the master.

To address problems those are specified above we design combination of task based sensing and critical event monitoring which in turn reduces power consumption and network energy is saved based on sleep and wake up techniques.

\section{Methodology}

In this section we discuss about our propose methodology.

We have considered 2 slave nodes but we can use $n$ number of nodes depending on the application. Our evaluation configuration consists of a connected receiver to a PC surrounded by $\mathrm{n}$ nodes of sensors. The embedded kit developed has PIC18F4520 microcontroller, temperature sensor, and fire sensor, potentiometer to indicate various energy level voltage, gas sensor and RF module. The on board sensor collects data from surrounding environment and with the help of UART this data is fed to controller, further the controller compiles a frame for communication adding ,,source ID $^{\text {ee }}$ and „,destination ID ${ }^{\text {ee }}$, which help to direct the data at the destination. Both the slaves are identical.

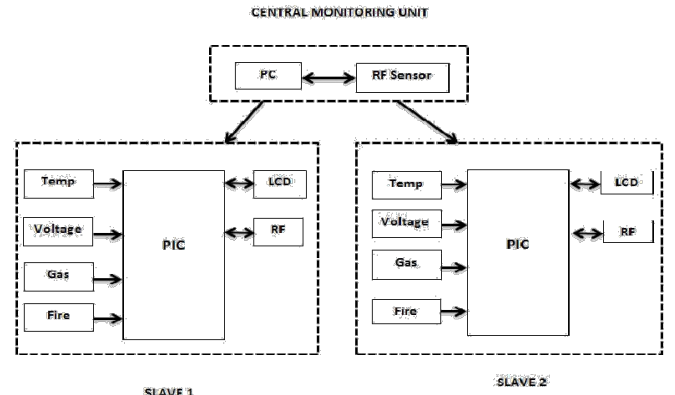

Figure 2: System Implementation 
Here we are reconfiguring the WSN using RF sensors. We are considering 1 Master and 2 slave's structure. In this network we are using the combination of task based sensing and critical event monitoring to reduce the power consumption of the network.

We are using 2-stage protocol as sensor nodes use two types of messages

\section{REQUEST Phase}

Respond (DATA to communication) Phase

REQUEST Phase: In this phase the Base station sends a request to Sensor Node containing the Master ID, Request ID. Sensor node after receiving the frame compares ID to its Own and sends the Data to master. In the cluster the same process is repeated. The cluster Head sends a Request to both the Slaves for data.

RESPONSE Phase: In this phase the Sensor node after receiving the frame compares the ID to its Own and sends the Data of both the slaves to master. The Response frame contains the Master ID, Response ID and the sensor data.

As shown in the figure 2 we are implementing the Task based sensing. Many applications in WSN have redundant data, which means that the data does not change very fast. A task is characterized by different parameters: type of sensing, number of sensing operations, period of sensing and the intended nodes (nodes required to sense data). So sending the same data again and again results in Wastage of Network energy which in turn reduces network life time.

So, here in our project we will send the data whenever the sensor crosses a threshold /Set point. That means whenever a new data is present, then only the slave will send the data frame in response. This results in less number of Communication frames, which increases the Network efficiency.

Also, we are implementing the Adaptive power transmission based on Battery voltage. As soon as the sensor node energy level reduces, we reduce the request time of the network to increase the network life. So, the communication between slave and master depends on the energy levels which results in higher efficiency.

Also we are designing a PC based GUI through which we can monitor the WSN sensor data.

\section{RF Power Consumption in Sleep and Wake up States}

The goal of sleep and wake-up state is to force the nodes to sleep when they are inactive while performing specific activity. There are two types of sleep states periodic sleep and continuous sleep.

- Periodic Sleep: When there is no task the nodes are placed in periodic sleeping, where after certain period they wake up and again go to sleep states. This alternation between sleep and wake-up states is known as periodic sleep.

- Continuous Sleep: The nodes may go into sleep state between two sensing actions and then wake up to sense and then again sleep purposefully. This is known as continuous sleep state. This continuous sleep can be very immense if the detection period is in hours or days.

We are using 2 conditions normal and adaptive power mode. When the battery voltage is below 10 volts the sensor nodes are in adaptive power mode and when it is greater than 10 volts then the sensor nodes are in normal power mode. During transmission, power which we require to send the data is more. So we are reducing the transmission frames to increase the power as well as efficiency of the network.

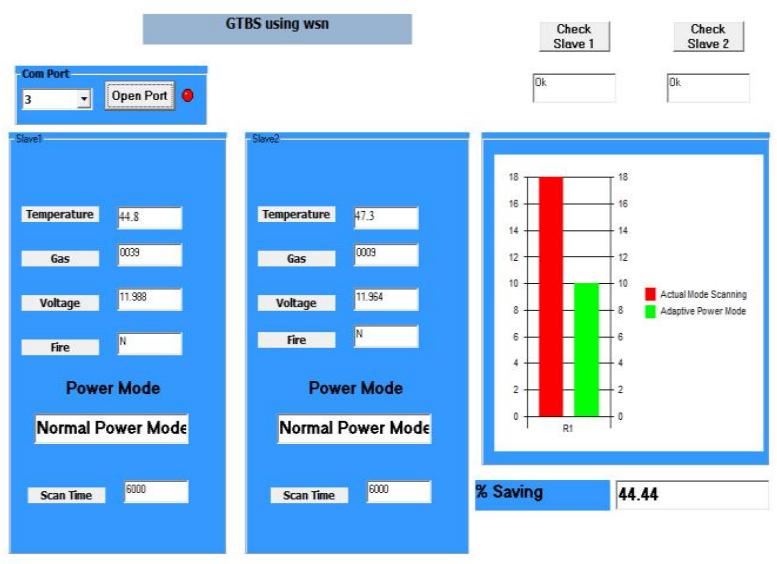

Figure 3: When Both the Slaves are in Normal Mode

In figure 3. We have considered that both the slaves are in normal mode and accordingly it is transmitting the response frames. Red colour indicates actual scanning response transmission frames and green colour indicates 
the adaptive power transmission frames. And to check whether slaves are in proper condition we check it by giving some pilot signal that is shown in above figure.

By setting the temperature, gas at a particular set point our new data will transmit as explained in the above algorithm. Among any of three sensors if any one sensor crosses its threshold point then $\mathrm{n}$ there it will transmit the data to master.

Slave 1 and 2 in above fig is for monitoring purpose. We are discussing about the transmission response frames which are indicated in green and red colour. In normal mode actual transmission response frames are 18 and adaptive power mode transmission frames are 10 because new data is detected to transmit the frames.

So per-cent saving is

$((18-10) / 18) * 100=44.44 \%$

The scan time shown is 6000 which means that after every $2 \mathrm{sec}$ delay it will sense the data.

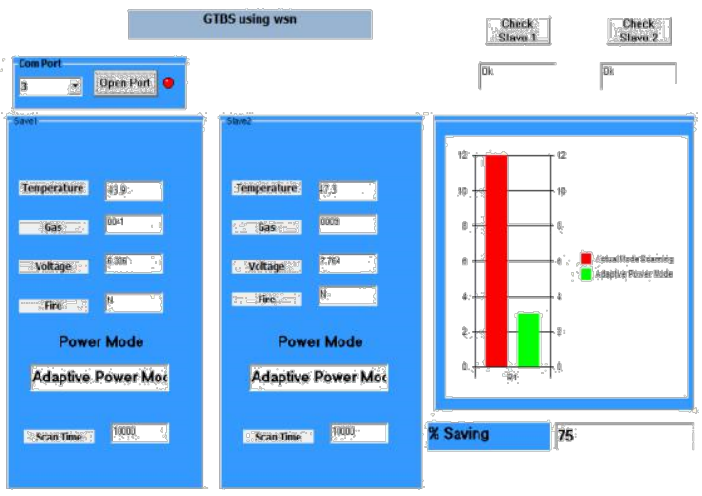

Figure 4: When Both the Slaves are in Adaptive Power Mode

In the above figure both the slaves are in adaptive power mode. So number of frames as compared to normal mode is less. Battery voltage is below 10 volts. And our scan time to sense the data of every sensor is $10000 \mathrm{sec}$. so after every $10 \mathrm{sec}$ it will sense the data and accordingly transmit. Here the actual transmission frames are 12 and adaptive power transmission response frames are 3.

So per-cent saving is

$((12-3) / 12) * 100=75 \%$

According to above results obtained we can specify the power consumption as follows
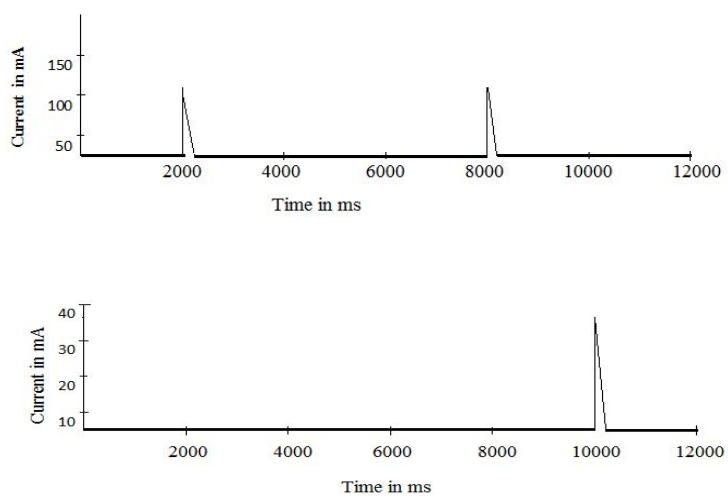

Figure 5: Sleep and Wake up States of the Sensors during Normal and Adaptive Power Mode

The frames will be transmitted as follows. Table I Shows the transmitted frames during sleep and wake up states

\begin{tabular}{|l|l|l|}
\hline Sensors & Transmitted frames during Normal Mode & Transmitted frames during Adaptive Power Mode \\
\hline Sensed by temp & $2.1 \mathrm{sec}$ & $10.1 \mathrm{sec}$ \\
\hline Sensed by gas & $4.2 \mathrm{sec}$ & $20.2 \mathrm{sec}$ \\
\hline Sensed by fire & $6.3 \mathrm{sec}$ & $30.3 \mathrm{sec}$ \\
\hline Sensed by temp & $8.4 \mathrm{sec}$ & $40.4 \mathrm{sec}$ \\
\hline Sensed by gas & $10.5 \mathrm{sec}$ & $50.5 \mathrm{sec}$ \\
\hline Sensed by fire & $12.6 \mathrm{sec}$ & $60.6 \mathrm{sec}$ \\
\hline
\end{tabular}


In normal condition the scan time for detecting any individual data is $2 \mathrm{sec}$ and transmission of frame is $0.1 \mathrm{sec}$ because 100 milli sec $=0.1 \mathrm{sec}$. Therefore time to send one frame is $2.1 \mathrm{sec}$. After every $6 \mathrm{sec}$ approximately new data is received to master. Here we are using periodic sleep state so that the sensor goes into the sleep for certain period and again wake up for some period.

In adaptive power mode condition the scan time for detecting any individual data is 10 sec. and the transmission response frame is $1 \mathrm{sec}$. Therefore to send one frame is about $10.1 \mathrm{sec}$. After every $10 \mathrm{sec}$ new data is received to the master.

From the above fig 5 we observe that the efficiency during the adaptive mode is increased up-to $50 \%$. Because the in $10 \mathrm{sec}$ we are sensing one frame during adaptive power mode and 2 frames in normal power mode so $1 / 2 * 100=50 \%$.

\section{Evaluation Results}

In this section we represent power consumption of nodes compared with conventional (normal mode) and gtbs mode, event delivery ratio and the graph that is based on various battery voltages and also mentioned about critical event monitoring which saves the overall lifetime of network.

To calculate the power of nodes and to compare our system with conventional (traditional) task based system we measured the current during normal mode and current during green task base sensing by using multi-meter. The current during normal mode is 104.23 milli amperes and during gtbs mode current is 35.25 milli amperes. To calculate power the formulae is given by $\mathrm{P}=\mathrm{V} * \mathrm{I}$. here the voltage is fixed 5 volts. Accordingly we will calculate power for min, hour, day, month and year.

Table II shows Power consumed in gtbs mode and normal mode.

\begin{tabular}{|c|c|c|}
\hline & Normal Mode & Gtbs Mode \\
\hline $1 \mathrm{sec}$ & 0.52175 & 0.17625 \\
\hline $1 \mathrm{~min}$ & 31.305 & 10.575 \\
\hline 1 Hour & 1878.3 & 634.5 \\
\hline 1 day & 45079.2 & 15228 \\
\hline 1 Month & 1352376 & 456840 \\
\hline 1 Year & 16228512 & 5482080 \\
\hline $\begin{array}{r}18000000 \\
16000000 \\
14000000 \\
12000000 \\
10000000 \\
8000000 \\
6000000 \\
4000000 \\
2000000 \\
0\end{array}$ & hour 1 day $\frac{1}{\operatorname{month}^{\prime}} 1$ vo & $\begin{array}{l}- \text { Conventional Mode } \\
=\text { Gtbs Mode }\end{array}$ \\
\hline
\end{tabular}

Figure 6: Graph of the Gtbs Mode vs Conventional Mode

Thus figure 6 shows energy consumption is more as compared to traditional task base sensing. The overall energy consumption that is given by conventional mode-gtbs/ conventional mode which is $66.21 \%$ approximately.

As we have used combined concept of critical event monitoring and task base sensing schemes percent saving of energy is more in the critical event monitoring by considering assumption as follows

For e.g.) A gas station has been found leakage for 2 hours the sensors receives the data continuously for two hours in gtbs mode so communication is initiated for 2 hours for rest hours it will be in normal condition.

Among 24 hours we have 2 hours critical event monitoring we calculate the \% saving, assuming data from table 1

$634.5 * 2=1269$

normal mode power consumed of $24 \mathrm{hrs}$ - gtbs power consumed for two hrs

$45079.2-1269=43810.2$

$43810.2 / 45079.2 * 100$

$97 \%$ 
Energy being saved is about $97 \%$ approximately.

Fig shown below is of Event Delivery Ratio(EDR) plotted as transmission frames vs event delivery ratio in \% for two sensor nodes.

EDR is defined as

$\mathrm{EDR}=$ received packets $\div$ sending request $*$ Intended nodes

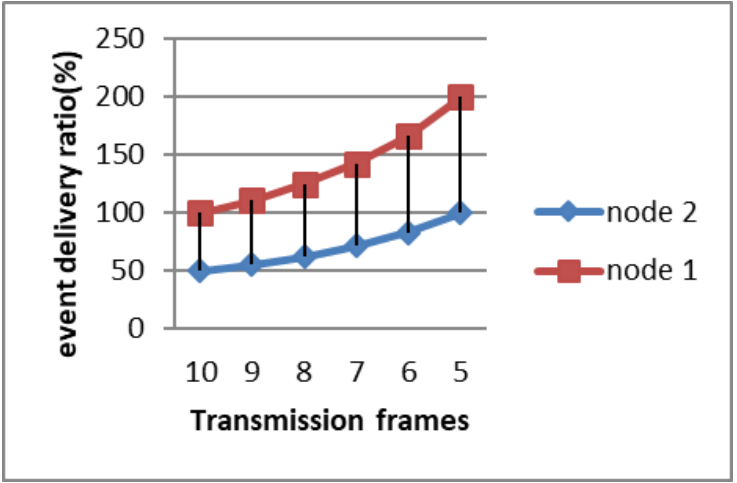

Figure 7: Event Delivery Ratio Graph for 2 Sensor Nodes.

During the conventional mode there is some problem in transmitting the data because of losses in transmission packets and interference. Transmission power required to transmitt data is more which will influence on the quality of links in the network. So if we reduce the transmission frames we increase the efficiency.

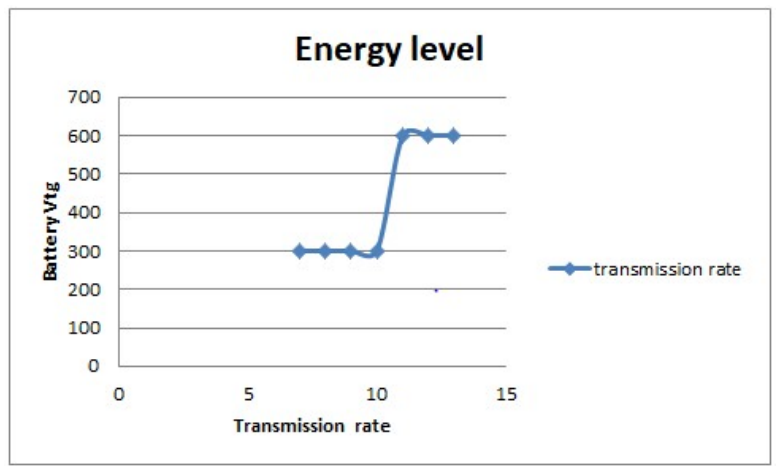

Figure 8: Various Battery Energy Levels Graph

Figure 8 shows the graph plotted as battery voltage verses transmission rate. Sensors continuously sense the data so the sensor energy is degraded. As the energy reduces we reduce transmission frames or request time. From the figure 8 we can see that as we have set the battery voltage at particular set point that is below $10 \mathrm{~V}$ in adaptive and above $10 \mathrm{~V}$ normal power modes. As the battery voltage is decreasing the transmission frames are also reduced. Hence we can say that the communication between master and slave is based on battery energy levels to increase the network lifetime.

\section{Conclusion}

The green task base sensing scheme is a task based sensing that not only prevents wasting of power in unnecessary signaling but also utilizes techniques for achieving reliable and energy efficient WSN. As we reduce the transmission response frames our energy is saved up to $50 \%-70 \%$ respectively as compared to conventional task base sensing. Moreover, we obtain the increment during critical event monitoring to increase the overall energy efficiency of network up to $97 \%$ based on our assumptions. This system permits the WSN to sense data in the form of events directed to sensor nodes. In green task based sensing it depends on the industry or the applications. Suppose if the gas leaked for only $5 \mathrm{mins}$ in the industry so the task is being initiated only for 5 mins. So our energy is being saved. In future, we intend to expand scalability analysis to include variation of largest number of nodes to maintain excellence of our proposed scheme.

\section{References}

[1] A. Prayati, C. Antonopoulos, T. Stoyanova, C. Koulamas, and G. Pa padopoulos, "A modeling approach on the TelosB WSN platform power consumption,” Journal of Systems and Software, vol. 83, no. 8, pp. 1355$1363,2010$. 
[2] A. Tripathi, N. Yadav, and R. Dadhich, "Secure-spin with cluster for data centric wireless sensor networks," in 2015 Fifth International Conference on Advanced Computing and Communication Technologies (ACCT). IEEE, 2015, pp. 347-351.

[3] I. F. Akyildiz, W. Su, Y. Sankarasubramaniam, and E. Cayirci, "Wireless sensor networks: a survey," Computer Networks, vol. 38, no. 4, pp. 393-422, 2002.

[4] M. Tahir, N. Javaid, A. Iqbal, Z. A. Khan, and Alrajeh, "On adaptive energy-efficient transmission in WSNs," International Journal of Distributed Sensor Networks, vol. 2013, no. 10, p. 10, 2013.

[5] D. Son, B. Krishnamachari, and J. Heidemann, "Experimental study of the effects of transmission power control and blacklisting in wireless sensor networks," in IEEE Communications Society Conference on Sensor and Ad Hoc Communications and Networks (SECON'14). IEEE, 2004, pp. 289-298.

[6] M. Balakrishnan, E. E. Johnson, and H. Huang, "TEAN-Sleep for Distributed Sensor Networks: Introduction and _-Metrics Analysis," in IEEE Military Communications Conference (MILCOM ${ }^{\text {ce } 07) . ~ I E E E, ~}$ 2007, pp. 1-7.

[7] W. Ye, J. Heidemann, and D. Estrin, "Medium access control with coordinated adaptive sleeping for wireless sensor networks," IEEE/ACM Transactions on Networking, vol. 12, no. 3, pp. 493-506, 2004

[8] B. Jiang, B. Ravindran, and C. Hyeonjoong, "Probability-based prediction and sleep scheduling for energyefficient target tracking in sensor networks," IEEE Transactions on Mobile Computing, vol. 12, no. 4, pp.735-747, 2013.

[9] S. Charalambos and V. Vasos, "Source-based routing trees for efficient congestion control in wireless sensor networks," in IEEE $8^{\text {th }}$ International Conference on Distributed Computing in Sensor Systems (DCOSS). IEEE, 2012, pp. 378-383.

[10] Xu, X., Luo, J. and Zhang, Q. (2012) „Delay tolerant event collection in sensor networks with mobile sink $^{\text {ee }}$, Proceedings of The 29st Annual IEEE International Conference on Comp Communications (INFOCOM 2010), 15-19 March, San Diego, CA, USA.

[11] A. S. Weddell, N. R. Harris and N. M. White, "Alternative Energy Sources for Sensor Nodes: Rationalized Design for Long-Term Deployment," 2008 IEEE Instrumentation and Measurement Technology Conference, Victoria, BC, 2008, pp. 1370-1375.

[12] K. Phan, R. Fan, H. Jiang, S. Vorobyov, and C. Tellambura, "Network lifetime maximization with node admission in wireless multimedia sensor networks," IEEE Transactions on Vehicular Technology, vol. 58, no. 7, pp. 3640-3646, September 2009. 